\section{IDENTIFICANDO LOS LIMITANTES DE GENERACIÓN DE EFECTIVO: BASES PARA UNA METODOLOGÍA DE MEJORA CONTINUA*}

\author{
Igor Rivera \\ Instituto Politécnico Nacional - UPIICSA \\ Av. Té 950, Col. Granjas México \\ Del. Iztacalco, México, D.F., 08400 \\ iariverag@ipn.mx \\ Juan Morúa Ramírez \\ Université de Lorraine, Francia \\ 13 Rue Maréchal Ney, 54000 Nancy, France \\ j_morua@hotmail.com

\section{IDENTIFYING THE CONSTRAINTS ON CASH GENERATION: BASIS OF A METHODOLOGY FOR CONTINUOUS IMPROVEMENT}

\begin{abstract}
* Agradecemos el apoyo otorgado para el desarrollo de este artículo al Instituto Politécnico Nacional, Instituto Tecnológico de Tantoyuca y Secretaría de Ciencia, Tecnología e Innovación del D.F. México (ICYTDF/325/2011).
\end{abstract}

Cómo citar este artículo/ Citation: Rivera, I. y Morúa Ramírez, J. (2013). Identificando los limitantes de generación de efectivo: bases para una metodología de mejora continua. Arbor, 189(760):a016. doi: http://dx.doi.org/10.3989/ arbor.2013.760n2002

Recibido: 29 abril 2010; Aceptado: 18 marzo 2011.

RESUMEN: Dentro de las organizaciones encontramos limitantes que hacen del proceso de generación de efectivo un largo y penoso camino. Nuestro objetivo en este artículo es hacer una revisión teórica sobre los limitantes de generación de efectivo (LGE), desde el proceso de transformación hasta el valor añadido. Esta revisión establece un punto de vista diferente a aquella establecida por la teoría clásica del management y está enfocada a las pequeñas y medianas empresas (Pymes) de manufactura que no sean de reciente creación. Finalmente, el escrito menciona pautas para proponer una mejora continua, con respecto a la generación de efectivo: El análisis retrospectivo y la identificación del proceso crítico; La definición de parámetros clave y el pilotaje estratégico; La identificación dentro del proceso crítico del principal elemento que limita la generación de efectivo; La definición de un plan de acción para poder reducir, controlar o eliminar el limitante de generación; El establecer los pasos anteriores como un ciclo continuo.

PALABRAS CLAVE: Limitantes de generación de efectivo; incertidumbre; variabilidad; restricción; mejora continua.
Copyright: (C) 2013 CSIC. Este es un artículo de acceso abierto distribuido bajo los términos de la licencia Creative Commons Attribution-Non Commercial (by-nc) Spain 3.0.

ABSTRACT: Inside organisations we always find constraints which the cash generation process a long and tortuous path. Our aim in this article is to offer a theoretical review of these constraints, from the process of transformation through to value added. This review takes a different point of view from classical management, focusing on small and medium-sized already established manufacturing organisations. Finally, the article mentions guidelines, with regards to cash generation, which propose continuous improvement: retrospective analysis and identifying the critical process; Defining key parameters and strategic piloting; Identifying the main element which limits cash generation within the critical process; Defining an action plan to reduce, control or eliminate the constraints on generation; Establishing the previous steps as a continuous cycle.

KEYWORDS: Cash generation constraints; uncertainty; variability; constraints; continuing improvement. 


\section{INTRODUCCIÓN}

La inquietud de identificar a los limitantes de generación de efectivo, ha surgido de nuestro proceso de investigación tendiente a encontrar aquellos elementos que permitan a las pequeñas y medianas empresa (Pymes) mejorar su desempeño. La idea parte de estudiar cómo se aumenta el efectivo dentro de las pequeñas y medianas empresas, para después profundizar en el proceso de generación de efectivo. El reto es identificar cuáles son aquellos factores que limitan este proceso, partiendo de la hipótesis inicial siguiente: si la empresa establece una metodología de mejora continua para eliminar o reducir los efectos de los limitantes de generación de efectivo obtendrá una mejora en el desempeño, no solo en los parámetros financieros, también en los parámetros logísticos, de servicio y calidad, de innovación, etc. Por lo que el primer paso que daremos es la identificación de estos limitantes de generación.

La diversidad de tipos de empresa, nos hace enfocar esta aportación teórica hacia las empresas pequeñas y medianas dedicadas a la manufactura que han pasado la prueba del tiempo y se han logrado mantener en la competencia en un mercado específico.

Dentro del presente artículo nos basaremos en la idea de que el aumento del efectivo es uno de los principales retos objetivos dentro de las Pymes, así, con esta base desglosaremos el concepto de generación de efectivo, que forma parte del aumento de efectivo, y mediante este análisis estableceremos los elementos clave que forman parte de la generación, para por último establecer de manera general, los elementos que limitan la generación de efectivo.

\section{LA EMPRESA COMO SISTEMA COMPLEJO}

Antes de empezar con el presente artículo y tomando como base la teoría de sistemas, queremos enfatizar que la empresa es un sistema complejo, con diferentes interacciones tanto dentro de la misma, como en los sistemas superiores que lo rodean, tales como la sociedad y el mercado. A su vez en ella convergen sistemas humanos y tecnológicos, se crea cultura, existen relaciones de poder entre sus integrantes, y un sinnúmero más de elementos en constante cambio.

Toda estas interrelaciones hacen de la empresa un sistema complejo (Barba - Solís 1999), por lo que antes de analizar a los elementos que limitan la generación de efectivo, que es el reto dentro del presente trabajo, debemos de empezar identificando a los sistemas superiores que influyen en su desempeño.

\section{La influencia de sistemas superiores}

Al ser la empresa un sistema complejo, existen elementos que afectan su desempeño para alcanzar la meta de generación de efectivo. Estos sistemas son referidos a sistemas superiores (Blanchard, 2004) tales como el sistema económico, el mercado internacional, el sistema financiero nacional, etc. y también elementos propios de la naturaleza que ejercen una influencia hasta cierto punto incontrolable por parte de la empresa (Becker, 2003). Estos factores del medio ambiente están más relacionados con la incertidumbre dominante (Marckus, 2003), en donde la empresa no puede influir ni controlar. Así podemos encontrar un número ilimitado de factores de influencia de sistemas superiores, sin embargo todos agregan un factor importante: la incertidumbre.

Mendez (2002) define a la incertidumbre como la duda en torno a cómo se van a desenvolver los acontecimientos en el tiempo y que pueden afectar positiva o negativamente el desarrollo empresarial, así un desastre natural puede terminar con una empresa exitosa y al mismo tiempo elevar la demanda de productos de otra. En este tipo de eventos, la empresa no tiene control, además de que es difícil predecirlos, por lo que algunas organizaciones grandes y gobiernos, realizan intentos al aplicar el business continuity management (Hiles, 2007), que es una metodología para hacer frente a posibles desastres y poder continuar con la operación de negocios, pero para las Pymes es muy difícil poder destinar recursos para establecer este tipo de programas de prevención.

Es importante dentro de este escrito, el clasificar a la incertidumbre, sobre la cual las Pymes pueden tomar acciones más fácilmente, para lo cual tomaremos la clasificación que realiza Markus (2003): la incertidumbre según su grado de información y posibilidades de que sucedan los eventos pueden ser: (1) riesgo en donde los posibles resultados y sus probabilidades son conocidas, (2) incertidumbre base, en donde los posibles resultados son conocidos en principio, pero las posibilidades solo pueden ser subjetivamente estimadas y (3) la incertidumbre dominante, donde los posibles resultados y las posibilidades de que sucedan no son conocidos.

Otros elementos del medio ambiente, también influyen en las empresas, estos son propios del elemento humano y los mencionaremos a continuación.

\section{Sociedad y cultura}

La ideología de una sociedad, referida a los diferentes tipos de ideas, conocimientos y creencias que integran un orden social (Bourguignon et al, 2004) y que dirigen aspectos de la vida como la moral o inclusive las metas personales. Es una fuente importante de influencia en el desempeño de las organizaciones. A su vez Savall (2008) menciona que los conceptos culturales afectan en gran modo el desempeño de una empresa, así por ejemplo, entre dos empresas iguales en 
cuanto a tecnología y capital, la que desarrolle mejor sus aspectos de cultura organizacional será la que obtenga mejores resultados. Estos aspectos sociales son importantes de mencionar por la fuerza que ejercen sobre la empresa y que afectan a los procesos (estratégico, de soporte y de generación) que la componen.

\section{Mercado Global}

Segrestin (2004) enfatiza en su estudio, la entrada a partir de 1980 de un nuevo paradigma dominante en las ciencias de la gestión, originado principalmente por la economía global, motivo por el cual los paradigmas anteriores quedaron obsoletos, así se han creado nuevas formas de organización orientadas a la calidad, a la logística, al desarrollo de aspectos culturales, a la reinvención de los procesos, entre otras.

La influencia de los sistemas superiores y el constante cambio en el paradigma en las ciencias de la gestión, nos hace proponer el utilizar el aumento de efectivo como principal elemento de monitoreo, para evaluar y dar origen a los cambios dentro de las Pymes. Para lograr este objetivo debemos revisar a lo que llamamos aumento de efectivo.

\section{EL AUMENTO DE EFECTIVO Y LA GENERACIÓN DE EFEC- TIVO}

Al establecer el aumento de efectivo como principal meta dentro de la vida de la empresa convierte un concepto simple en todo un esfuerzo, porque para identificar que existió aumento de efectivo dentro de un periodo determinado es necesario que haya existido: a) flujo de efectivo generado por la empresa (pro-

Figura 1. Aumento de efectivo. Basado en Rivera y Morúa 2010

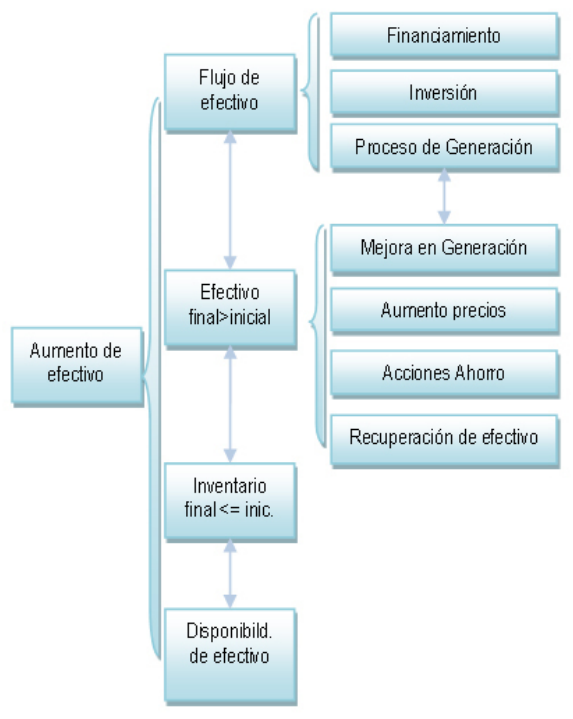

ceso de generación de efectivo), creado por financiamiento con terceros y por inversiones, b) el efectivo final sea mayor al efectivo inicial del periodo, c) los inventarios finales dentro de la empresa sean menores o iguales a los iníciales (a menos que el crecimiento de inventario responda a una estrategia del negocio) y por último d) que exista disponibilidad de efectivo (Rivera y Morúa, 2010).

Partimos de la hipótesis de que las condiciones para el aumento de efectivo, se vuelven más complicadas entre más crece la empresa. En la figura 1, podemos observar de forma esquemática estas condiciones, en donde se requiere el establecimiento de acciones estratégicas específicas adecuadas al momento que se encuentra la empresa. Así, por ejemplo, una empresa, puede ser que requiera de financiamiento para aumentar su flujo de efectivo, y establecer acciones de ahorro dentro de la empresa para lograr que el efectivo final de un periodo sea superior al efectivo inicial.

La importancia del estudio del aumento del flujo de efectivo mediante el proceso de generación, es que este representa el mayor porcentaje de obtención de recursos (Mackevicius, 2006), así mediante la ley de Pareto, nos enfocaremos a este proceso. Analizando el proceso de generación de efectivo dentro de las Pymes de manufactura, observamos que este es el resultado de la interacción de tres elementos clave (Rivera y Morúa, 2010) el proceso de transformación, el flujo de materiales e información y la creación de valor.

Como siguiente paso para poder lograr nuestro objetivo, debemos de profundizar en los conceptos de transformación, flujo y valor. Por lo que nuestra siguiente tarea es desglosar los elementos importantes de cada uno de estos procesos que pueden llegar a limitar el proceso de generación de efectivo.

\section{Procesos claves para la comprensión de la empresa}

Antes de continuar debemos de especificar qué es un proceso, para lo cual vamos a citar a Segrestin (2004) que identifica un proceso como cualquier serie de actividades que producen un resultado representado en valor para un cliente, además clasifica los procesos dentro de las empresas en: 1) los estratégicos que se ocupan tanto de la competitividad de la empresa como del desarrollo e innovación, 2) el proceso de la cadena de operación, el cual permite la generación de efectivo dentro de la empresa y 3 ) los procesos de soporte que se pueden desarrollar tanto dentro de la empresa como por medio de un proveedor de servicios y que permiten la cohesión y aseguran la continuidad de los otros dos principales procesos.

Esta identificación de tres grandes procesos dentro de la empresa, nos permite profundizar en su estudio, sobre todo el que nos ocupa dentro del presente tra- 
bajo que es el proceso de la cadena de operación, la cual llamaremos en nuestro caso, el proceso de generación de efectivo. Pero como estos no se encuentran aislados, más bien interrelacionados debemos revisar cada uno aunque sea brevemente.

\section{Proceso estratégico: principal determinante del desempeño}

El proceso estratégico, bajo nuestra consideración, debe de integrar las actividades enfocadas a los siguientes aspectos: a) el aspecto competitivo de la empresa, b) la identificación, medición y coordinación de acciones referidas a los limitantes de generación de efectivo (motivo del presente trabajo) c) la interacción con actores externos d) y la innovación-diseño en su amplio sentido.

a) Aspecto competitivo. La actividad estratégica referido al aspecto competitivo, ha sido una de las más estudiadas e inclusive explotadas comercialmente durante los últimos años, esta actividad debe de estar enfocada hacia el desarrollo competitivo dentro del mercado con decisiones de segmentación de mercados, portafolio de productos, entre otros.

b) Identificación, medición y coordinación de acciones referidas a los limitantes de generación de efectivo. Este proceso está relacionado con dos aspectos estratégicos primordiales: el análisis retrospectivo y el pilotaje estratégico (Wegmann, 1999). Una de las primordiales funciones de la estrategia es el de realizar un análisis retrospectivo del estado de la empresa (Wegmann, 1999) estableciendo y analizando parámetros relacionados con la actividad pasada. La segunda acción, no menos importante, es el de establecer un pilotaje estratégico, es decir conducir a la empresa a un futuro deseado al tomar acciones para corregir y mejorar mediante la implementación de parámetros tanto subjetivos como objetivos.

c) La interacción con actores externos. Es referida a las actividades que se deben de realizar estratégicamente para que elementos tales como el desarrollo de actividades relacionadas con el medio ambiente, aspectos sociales, caritativos e inclusive legales, sean llevados a cabo para conservar, entre otras cosas, una buena reputación de la empresa, que permita la continuidad de la misma dentro de una sociedad.

d) Innovación-Diseño. Dentro del proceso de transformación, la función de innovación-diseño integra tanto las necesidades del cliente, en cuanto a la satisfacción de sus requerimientos, como a facilitar la producibilidad del producto, es decir la factibilidad de su producción y la adaptación, tanto a la estrategia del negocio correspondiente a la manufactura, como a los procesos de transformación y maquinaria y equipo que la empresa cuenta. El proceso de plasmar la idea de un diseño a un producto final requiere, además de una factibilidad de procesos de transformación, mantener presente una idea constante en el flujo del material, tomando en cuenta el diseño logístico de la empresa mantiene, Mather (1989) menciona la necesidad de diseñar productos con un enfoque logístico.

Es tan amplio este tema del proceso estratégico, que no podemos abarcarlo dentro del presente artículo, por lo que dejaremos esta interesante discusión para seguir adelante con el propósito del presente escrito, por lo que resumiendo, el proceso estratégico es un elemento determinante en el desempeño de la empresa, dado que es el actor principal en la dirección de la misma, así como el principal motor en el establecimiento de la mejora continua.

\section{Procesos de soporte: cohesión y continuidad}

Los procesos de soporte son aquellos establecidos para lograr la cohesión y lograr la continuidad de la empresa, así elementos como la información, la contabilidad, la administración del recurso humano, entre otros, son necesarios.

En este escrito, tomaremos como ejemplo a los procesos de información, los cueles son determinantes en el buen funcionamiento de toda empresa. Al referirnos a los procesos de información, no sólo lo hacemos para tomar en cuenta a los procesos formales, sino también los informales, derivados de las relaciones de poder dentro de la empresa (Barba, 1997), los cuales mantienen una fuerte relación con la cultura organizacional de la empresa. Así hablamos de un sistema de información que permita la coherencia de las acciones, refiriéndonos a este sistema en su amplio aspecto, es decir tanto de la información de sus bases formales (ERP, MRP, etc.), la comunicación de políticas y procedimientos, y de las que originan y dan pie a los sistemas de medición del desempeño. Bajo esta amplia concepción de sistemas de información podemos situar a diferentes actividades, las cuales se les han encargado clásicamente a diversas áreas. Resumiendo podemos definir a los procesos de soporte como todas las actividades que se deberán de llevar a cabo para el aseguramiento de la continuidad del proceso de generación de efectivo.

\section{Proceso de la cadena de producción = proceso de ge-} neración de efectivo

Dentro del proceso de generación de efectivo identificamos tres componentes interrelacionados: 
- el proceso de transformación,

- el flujo de materiales y de información y

- la creación de valor añadido.

Así nuestra siguiente tarea es definir estos tres elementos. Basándonos en la afirmación anterior la generación de efectivo se basa en: a) trasformar las materias primas en productos terminados, b) que el flujo tanto de materiales como de la información relativa a ellos, desde la materia prima hasta la entrega de productos terminados al cliente sea llevado de manera óptima (involucrando las acciones de compra, almacenaje, venta, etc.) c) y que estos productos terminados y el servicio otorgado por la empresa representen un valor superior según la opinión del cliente, manteniendo una fuerte relación con dos elementos primarios que son tanto el tiempo como los inventarios.

El proceso de transformación: El proceso de transformación está referido al cambio físico o químico de una materia prima, este puede ser un proceso de estampado, una mezcla, un cambio de color, etc. a su vez incluye las relaciones hombre-máquina. Para poder identificar los limitantes de generación de efectivo es necesario profundizar en los diferentes estados clave referidos al proceso de transformación de las materias primas en productos terminados. Así el proceso de transformación involucra a la tecnología aplicada, la habilidad y capacidad técnica del capital humano.

Flujo de materiales y de información: El concepto de flujo ha sido utilizado desde Henrry Ford y explotado por el sistema de producción Toyota (Koskela, 2000) y ha dado pautas para optimizar los flujos mas allá de la empresa, involucrando a clientes y proveedores en la administración de las cadenas de suministro (Lambert - Cooper, 2006).

Al hablar de flujo, no solo nos referimos al movimiento de los materiales, también involucra conceptos logísticos como: la administración de transporte y fletes, almacenamiento, manejo de materiales, administración de órdenes de producción, diseño de cadenas logísticas, manejo de inventarios, planeación de la demanda, administración de proveedores, compras y abastecimientos, planeación de la producción, programación de la producción, empaque y ensamble, y servicio al cliente. Estas se encontrarán dependiendo de los requisitos de la empresa (CSCMP, 2009).Además, la logística está involucrada en todos los niveles de planeación y ejecución estratégica ya que integra funciones las cuales coordina y optimiza como mercadotecnia, ventas, manufactura, finanzas e información tecnológica.

Valor añadido: El valor total de un producto es la suma tanto de un valor económico como de un va- lor añadido que es una percepción subjetiva (Rivera y Morúa, 2010), así se conjuntan tanto las características físicas del producto, las condiciones de entrega y tiempo de respuesta, el servicio, la atención que recibe un cliente por un producto, etc. Recordemos que el presente artículo es referido a las empresas de manufactura. Por lo que para aumentar el valor añadido de un producto, no sólo se deben de establecer productos que mantengan una calidad constante, referida a la repetitibilidad y reproducibilidad del producto, sino que también involucra un concepto más amplio referido a la creación de un sistema de cultura dentro de la empresa, enfocado a la calidad y el servicio.

Uno de los principales problemas de la precepción del cliente hacia el valor añadido es que este no se mantiene con las mismas características, conforme al tiempo. La estandarización de las características de un producto o servicio o la imitación de la competencia, hacen que lo que en el pasado fue un producto o servicio con alto valor añadido, sea convertido en la actualidad como una exigencia por parte del cliente. Esta característica del valor añadido hace que sólo el elemento humano sea creador de valor añadido, porque es el que con ideas, esfuerzo, creatividad y servicio logra atraer la preferencia de clientes. Es por lo anterior que el concepto de valor involucra cada vez más la creación de una cultura organizacional y el desarrollo de un capital intelectual.

Así, los procesos de transformación-flujo-valor añadido se encuentran interrelacionados (Koskela, 2000), y por ejemplo, algunas empresas de servicio mantienen una fuerte relación entre el flujo y el valor, en cambio para algunas empresas de manufactura la base de su generación se encuentra entre los procesos de transformación y flujo, para algunas otras los procesos de transformación y la creación de valor, y obviamente se encontraran empresas en donde estos tres elementos sean imprescindibles para la generación de efectivo. Por lo que no podemos hablar de una sin mencionar al menos otra, por lo que en el presente trabajo identificaremos a estos procesos por pares (trasformación-flujo, trasformación-valor, flujovalor) para identificar los limitantes de generación de efectivo.

\section{Limitantes de generación de efectivo}

Los limitantes de generación de efectivo son elementos en donde la empresa puede ejercer cierta influencia para eliminarlos o para reducir sus efectos. Recordemos que el objeto de estudio en el presente trabajo son las empresas pequeñas y medianas de manufactura, que se han mantenido durante un tiempo en el mercado, es decir no es de nuestro interés el estudiar empresas de nueva creación, así, creemos conveniente analizar los tres elementos de generación de efectivo. Este análisis lo realizaremos en pa- 
res, ya que, como veremos más adelante, definen en cierto grado la forma en que la empresa compite en los mercados. A continuación se detallan estos pares de generación de efectivo.

\section{Transformación-flujo}

Empresas que realizan producciones en masa, o mantienen una línea de productos delimitada y definida, requieren enfatizar sus esfuerzos en estos dos elementos, por una parte en los procesos de transformación, referidas al uso de tecnologías utilizadas para realizar la de trasformación y las habilidades y conocimientos técnicos para realizarlos. Y por otra parte en los procesos de flujo, con dos vertientes (Comeli, 2007): las de flujo de Información, referidas al proceso de informar el inicio y fin de la activación de los procesos requeridos para satisfacer la orden de un cliente y al flujo de materiales, referidos a los limitantes de flujo de materiales dentro de toda la empresa.

Se observa la esfera de influencia de estos tres factores: la restricción se encuentra inmersa dentro del proceso productivo, regularmente representada por una restricción física, la variabilidad se encuentra en todas las partes del proceso e influye en el producto, y por último la incertidumbre que proviene de factores externos.

\section{Flujo-valor}

Las empresas cuyas características comunes en el mercado en donde desarrollan sus actividades y que necesitan mejorar las características flujo-valor como forma de competitividad, requieren innovar sus procesos enfatizando tanto en la administración de la cadena de suministro como en la creatividad e innovación para añadir valor tanto en el servicio como en el mejoramiento de los procesos logísticos.

Un ejemplo de este tipo de empresa es aquella que ha logrado fabricar rápidamente sus productos, y que mantiene sus procesos controlados y una mezcla relativamente baja en la gama de productos que maneja, como puede ser una embotelladora, la cual podría lograr un mejor desempeño si enfatiza sus esfuerzos en los procesos de flujo-valor. En este caso, y de manera general, los limitantes de generación en que se debe enfatizar para mejora son aquellos relativos a eliminar la incertidumbre del mercado en su amplio aspecto, y de la creación y mejoramiento de la cultura organizacional orientadas a la atención y servicio al cliente.

\section{Transformación-valor}

Un caso específico de las empresas que debe de enfatizar los procesos de transformación-valor, son aquellas que trabajan bajo proyecto y además que la construcción de estos proyectos involucren un producto único, novedoso y en el que el factor tiempo no sea necesidad primordial para el cliente, sin embargo que requiera de un alto grado de personalización y de comprensión de sus necesidades. Para este tipo de empresas el capital intelectual y las capacidades técnicas y de innovación de sus empleados son la principal fuente de competitividad.

\section{Definición de limitante de generación}

Desde nuestro punto de vista un limitante de generación de efectivo es la falta, ausencia o carencia de una característica técnica, una habilidad administrativa, una aplicación tecnológica, un recurso, una capacidad, etc. relacionada con los procesos de transformación-flujo-valor y que es relativamente crítica dentro de la empresa en un periodo de tiempo determinado, y que por falta de está, no se puede mantener una generación constante de efectivo.

Bajo este concepto, parece que todo en cierto momento dentro de la empresa puede ser un limitante de generación, como por ejemplo una máquina, que en un pasado ha sido suficiente para producir, pero en un presente se convierte en una limitante de generación, al no poder dar la capacidad suficiente al sistema, convirtiéndose en una restricción física.

En trabajos anteriores (Morúa, 2009), hemos llegado a la conclusión de que los principales elementos que afectan la generación de efectivo, con respecto al flujo de los materiales y al proceso de transformación, se pueden clasificar en tres:

a. La restricción.

b. La variabilidad.

c. La incertidumbre.

Se puede concebir un cuarto elemento, el cual no queremos clasificarlo como limitante, porque a la vez es el principal elemento que añade valor, pero también es creador de costos ocultos (Savalle, 2008). Estamos refiriéndonos al elemento humano, que juega un papel doble dentro de las organizaciones, en el primero ayuda al crecimiento y rentabilidad del negocio, con iniciativa, participación, ideas, innovaciones, etc. En el segundo, mal dirigido, mal informado, sin motivación añade costos ocultos, crea conflictos, inhibe el crecimiento, entre otras.

Cada limitante de generación, anteriormente clasificado y el elemento humano, adquieren una importancia mayor dependiendo del proceso de generación con el cual compite, así la identificación y administración de una restricción física tiene mayor peso en un proceso de generación basado en la transformaciónflujo, que en uno basado en transformación-valor.

\section{BASES PARA ESTABLECER UNA MetOdOlogía PARA LA MEJORA CONTINUA}

Haciendo una breve revisión de nuestros dos apartados anteriores, y bajo la premisa de buscar alcanzar el aumento del efectivo, observamos lo siguiente: 
a. Existen elementos que afectan el proceso de aumento de efectivo, que provienen de elementos exteriores a la empresa, los cuales no puede controlar y que regularmente su fuente es de sistemas superiores. De estos elementos destacan los siguientes: a) la incertidumbre dominante, como un tipo de incertidumbre bajo la cual la empresa no puede tener control; b) la sociedad y cultura de la empresa, la cual mediante elementos como la ideología y hace que la misma se enfrente a diferentes retos, dependiendo de la localidad en donde interactué, y c) el último elemento mencionado, y que ha provocado cambios en el paradigma de las ciencias de la gestión, que es la competencia global. La generación de efectivo forma parte primordial para el aumento del efectivo, aunque no es la única vía para incrementar el efectivo dentro de la empresa (ver figura 1). Consideramos que sí es la más importante, dado que encuadra la razón de ser de la empresa, y establece la forma en la cual compite dentro de su segmento de mercado. Así esta generación de efectivo se puede dividir en tres procesos: transformación, flujo y valor.

b. Dentro de la empresa existen tres grandes procesos: a) el proceso de generación de efectivo, b) el proceso estratégico y c) los procesos de soporte.

c. Los limitantes de generación de efectivo se pueden clasificar en tres grandes apartados: la restricción física, la variabilidad, y la incertidumbre (referida a aquella a cual la empresa pude tener información), y hemos agregado un cuarto elemento al factor humano.

Estos elementos los tomaremos como fundamentos - bases para la elaboración de una metodología de mejora continua, y como cita Koskela (2000, pág 22) "estos deben de proveer una guía para el tomador de decisiones y para la operación, deben de orientar a la acción y su aplicación debe de permitir mejoras en el desempeño".

La metodología a elaborar deberá de contemplar a la empresa como a un todo, si la meta de la empresa es el aumento de efectivo (tal y como proponemos) observamos que para tal efecto se deben de considerar cada uno de los elementos establecidos en la figura 1.

Los elementos fundamentales para el desarrollo de una metodología para la mejora continua deben de ser:

a. El análisis retrospectivo y la identificación del proceso crítico. b. La definición de parámetros clave y el pilotaje estratégico.

c. La identificación dentro del proceso crítico del principal elemento que limita la generación de efectivo.

d. La definición de un plan de acción para poder reducir, controlar o eliminar el limitante de generación.

e. El establecer los pasos anteriores como un ciclo continuo.

Describamos brevemente lo anterior:

Análisis retrospectivo: El análisis retrospectivo se basa en el diseño de una serie de indicadores que midan el comportamiento que mantuvo la empresa en un periodo anterior. Su base fundamental se encuentra en los parámetros financieros (Wegmann, 1999), así como la consideración tanto de la historia de la organización, como de su propia temporalidad (Barba 1997), analizando el estado y la situación en la cual se encuentra. El fin de esta etapa es la Identificación del proceso crítico, es decir, que para mejorar los procesos dentro de la empresa (estratégico-soporte-generación) en primer lugar debemos de identificar cual de los tres es el que se considera crítico. Bajo la línea que hemos seguido en este articulo, es por demás decir que nos estamos enfocando en el proceso de generación, aun así dentro de una metodología debemos considerar los tres.

La definición de parámetros clave y el pilotaje estratégico: la identificación del proceso crítico, debe de dar como resultado una serie de objetivos a alcanzar, los cuales deben de establecer una condición ideal deseada, para lo cual es necesario establecer una serie de parámetros clave que permitan a través de su constantemente monitoreo un pilotaje estratégico.

Identificación del limitante de generación de efectivo: Bajo el concepto de limitante de generación de efectivo definido anteriormente, es importante entonces establecer las bases para identificar cuando un elemento, máquina, proceso, método, técnica, etc. se convierte en un limitante. Este proceso de identificación está ligado directamente con el proceso de medición y control a través de un análisis retrospectivo (Wegmann, 1999) y con el proceso de toma de decisiones (pilotaje estratégico) (Wegmann, 1999).

Plan de acción: El establecer una serie de pasos para eliminar, reducir o controlar a los limitantes de generación de efectivo que se han detectado, define la orientación de todas las acciones dentro de la empresa. Esta puede ser a través de la aplicación de técnicas, herramientas o filosofías de producción que lo permitan, tomando en cuenta que la aplicación no se basa en implementar una técnica porque está de moda, más bien porque ha surgido de una necesidad que enfrenta la empresa. 
Ciclo continuo: por último, esta serie de pasos deberá de adecuarse para que surja como un proceso de mejora continua, es decir que debe de existir un avance medible y se debe de realizar en un proceso cíclico.

\section{CONCLUSIONES Y FUTURAS INVESTIGACIONES}

La mejora continua es un proceso cíclico. Si dentro de las empresas se trabaja para eliminar o reducir los efectos de los limitantes de generación de efectivo, mediante una metodología y bajo el enfoque de una mejora continua, se logrará mejorar el desempeño de la misma.

Queda pendiente para futuras investigaciones el desarrollo a profundidad de una metodología para el mejoramiento continuo dentro de las empresas, así como su implementación y validación práctica.

A su vez surge la inquietud de profundizar en el estudio del papel del elemento humano como creador de valor añadido, y del estudio de este mismo elemento.

\section{BIBLIOGRAFÍA}

Barba Álvarez, Antonio y Solís Pérez, Pedro C. (1997): Cultura en las organizaciones, México, Vertiente Editorial.

Blanchard, Benjamin (2004): Logistic Engineering and Management, NewYork, Prentice Hall.

Bourguignon, Annick; Malleret, Véronique \& Nørreklit, Hanne (2004): "The American balanced scorecard versus the French tableau du bord: the ideological dimension". Management accounting research, Vol.15, $\mathrm{n}^{\circ} 2$, Waterloo, 107-134.

CSMP : Council of supply chain management professionals, consultado el 04 diciembre 2009, disponible en: http:// cscmp.org/aboutcscmp/definitions.asp

Comelli, M. Fenies, P. \& Tchernev, N. (2008): "A combined financial and physical flow evaluation for logistics process and tactical production planning: Application in a company supply chain". International journal of production economics vol. 112 issue 1. Elsevier, 77-95.

Hiles, Andrew (2007): The Definitive Handbook of Business Continuity Management, London, John Wiley \& Sons, Ltd.
Koskela, Larry (2000): An Exploration Towards a Production Theory and its Application to Construction, Finland, Technical Research Center of Finland, VTT Publications.

Lambert, Douglas \& Cooper Martha (2000): Issues in supply chain management. Industrial marketing management vol. 29, Elsevier. 65-83.

Mackevicius, Jonas \& Senkus, Kastytis (2006): "The system of formation and evaluation of the information of cash flows". Journal of business Economics and Management. Vol. VII, No. 4, London, Taylor \& Francis 171-182.

Markus, Becket \& Thorbjørn, Knudsen (2003): "The role of routines in reducing pervasive uncertainty". Journal of business research No. 58, Elsavier, 746 -757.

Méndez Morales, José (2002): Economía y empresa, México, MacGraw Hill.

Mather, Hal (1989): Manufactura Competitiva. México, Prentice Hall.

Morúa Ramírez, Juan (2009): Guía de estrategias operacionales para incrementar el efectivo en las Pymes. Tesis de maestría. México, UPIICSA, IPN
Mula, Poler, Garcia-Sabater \& Lario (2006): "Models for production planning under uncertainty: A review", International journal of production economics No. 103, Vernon. 271-285.

Rivera Gonzales, Igor \& Morúa Ramírez, Juan (2010): "Reconstruyendo el enfoque de aumento y generación de efectivo", Contaduría y Administración, México, UNAM, 131-150.

Sallenave, Jean-Paul (1997): La Gerencia Integral. México, Norma.

Savall, Henr \& Zardet, Véronique (2008): "Le concept de coût-valeur des activités. Contribution de la théorie socioéconomique des organisations». Sciences de gestion. No. 64. Paris, SARL de Presse. 61-89.

Segrestin, Denis (2004): Les chantiers du manager. Paris, Armand Colin.

Wegmann, Grégory (1999) : Les Tableaux de Bord Stratégiques: Analyse comparative d'un modèle nord-américain et d'un modèle suédois, cahier de recherche 99.11, Paris, IAE de Paris - GREGOR. 